\title{
Analysis of Off-state Leakage Currents in Poly-Si FinTFTs with Wide Drain by Microwave Annealing
}

\author{
Hsin-Hui Hu, ${ }^{1 *}$ Chun-Lin Huang, ${ }^{1}$ Yao-Jen Lee, ${ }^{2,3}$ and Kun-Ming Chen ${ }^{2}$ \\ ${ }^{1}$ Department of Electronic Engineering, National Taipei University of Technology, \\ No. 1, Sec. 3, Chung-Hsiao E. Rd., Taipei, 10608, Taiwan, R.O.C. \\ ${ }^{2}$ Taiwan Semiconductor Research Institute, \\ No. 26, Prosperity Road I, Hsinchu Science Park, Hsinchu, 30078, Taiwan, R.O.C. \\ ${ }^{3}$ Department of Physics, National Chung Hsing University, \\ No. 145, Xingda Rd., Taichung, 402, Taiwan, R.O.C.
}

(Received February 21, 2019; accepted December 2, 2019)

Keywords: fin-like thin-film transistors, wide drain, longitudinal band-to-band tunneling (L-BTBT), lowtemperature microwave annealing (MWA)

In this study, polycrystalline silicon fin-like thin-film transistors (poly-Si FinTFTs) with a wide drain structure are fabricated. The off leakage current of poly-Si FinTFTs with various extended wide drain lengths $\left(L_{E X}\right)$ is investigated. As $L_{E X}$ increases, the longitudinal electric field at the intrinsic drift $/ \mathrm{N}+$ drain junction decreases and improves the off leakage current derived from longitudinal band-to-band tunneling (L-BTBT). The off leakage current of poly-Si FinTFTs at different temperatures is also analyzed to investigate the leakage mechanisms. For poly-Si FinTFTs with a small $L_{E X}(=0$ and $0.8 \mathrm{~mm})$, the weak dependence of leakage current on temperature indicates that the band-to-band tunneling (BTBT) is dominant. For poly-Si FinTFTs with a large $L_{E X}$ of $1.6 \mathrm{~mm}$, the strong dependence of leakage current on temperature and the weak dependence of leakage current on drain voltage indicate that trapassisted tunneling (TAT) is the dominant leakage mechanism. These results indicate that it is gate-induced drain leakage (GIDL), resulting from L-BTBT, that is effectively suppressed by increasing $L_{E X}$.

\section{Introduction}

Gate-induced drain leakage (GIDL) is the major component of the off-state leakage current in fin-like field-effect transistors (FinFETs) and increases standby power dissipation. ${ }^{(1,2)}$ In a three-dimensional (3D) structure such as a FinFET, although the transverse band-toband (T-BTBT) tunneling in the gate-to-drain overlap region can be suppressed by reducing the transverse electric field, the band overlap between the valence band of the body and the conduction band of the drain region enables substantial longitudinal band-to-band tunneling (L-BTBT) in the OFF state. ${ }^{(3)}$ Therefore, 3D transistors for low-power applications are severely limited by L-BTBT. Over the past few years, some attempts have been made to reduce L-BTBT. ${ }^{(4-8)}$ 
In our previous work, polycrystalline silicon fin-like thin-film transistors ((poly-Si FinTFTs) with a wide drain structures were simulated. ${ }^{(9)}$ Extending the wide drain can effectively suppress the longitudinal electric field near the drain and improve L-BTBT. Because of their electron mobility being higher than that of amorphous silicon (a-Si), poly-Si TFTs exhibit a high carrier mobility and therefore a fast transient response and a high current-driving capability, which are suitable for system-on-panel (SoP) or system-on-glass (SoG) applications. The suppression of the GIDL current in poly-Si TFTs has become an important issue. Channel length scaling can improve the carrier mobility of the poly-Si TFTs owing to the reduced numbers of grain boundaries in the channel. ${ }^{(10)}$ As the channel length decreases, however, the short-channel effect (SCE) becomes more pronounced. Three-dimensional multigate structures, ${ }^{(11-13)}$ such as double-gate, tri-gate, and gate-all-around (GAA) structures, as well as the low-temperature microwave annealing (MWA) method ${ }^{(14,15)}$ are known as to improve gate controllability and restrict dopant diffusion, respectively, and effectively suppress the SCE. In this study, tri-gate FinTFTs with various extended wide drain lengths $\left(L_{E X}\right)$ are fabricated and the off leakage current for the different structures is investigated. The devices at different temperatures are also analyzed.

\section{Materials and Methods}

All the poly-Si FinTFTs discussed in this study are fabricated on a 6" silicon wafer with a 1- $\mu$ m-thick wet oxide layer as the substrate. A 100-nm-thick undoped a-Si layer is deposited on the substrate by low-pressure chemical vapor deposition (LPCVD) as the active layer. After the solid phase crystallization (SPC) of the a-Si layer at $600{ }^{\circ} \mathrm{C}$ for $24 \mathrm{~h}$ in nitrogen ambient, the polycrystalline silicon layer is defined. The active region is then patterned by electronbeam lithography and transferred by reactive ion etching to form multiple nanowires (NWs). Thereafter, a 50-nm-thick tetraethyl orthosilicate (TEOS) layer is deposited by LPCVD as a gate insulator and a 100-nm-thick in situ $\mathrm{n}^{+}$poly-Si layer is deposited and patterned to form the gate electrode. Subsequently, the drift region and the self-aligned source and drain are implanted with $\mathrm{BF}_{2}{ }^{49+}$ at $20 \mathrm{keV}$ at doses of $5 \times 10^{14}$ and $5 \times 10^{15} \mathrm{~cm}^{-2}$, respectively. Dopants are activated by low-temperature MWA at $2400 \mathrm{~W}$ for $300 \mathrm{~s}$ below $550{ }^{\circ} \mathrm{C}$. Finally, a $300-\mathrm{nm}-$ thick TEOS oxide passivation layer is deposited followed by $\mathrm{Ti} / \mathrm{TiN} / \mathrm{AlSiCu} / \mathrm{TiN}$ contact metallization. The electrical characterization is carried out by on-wafer measurements using an Agilent 4155C semiconductor parameter analyzer and Agilent EasyEXPERT software.

\section{Results and Discussion}

\subsection{Poly-Si FinTFT structures}

Figure 1 shows schematic top views of the poly-Si FinTFTs with $L_{E X}=0,0.8$, and $1.6 \mathrm{~mm}$. The channel length $(L)$ is $0.7 \mathrm{~mm}$. The metal field plate is connected to the gate electrode as a gate field plate (GFP) and the drift region is undoped, which helps to improve the breakdown voltage by spreading the depletion region at the surface and lowering the electric field. Figure 2 


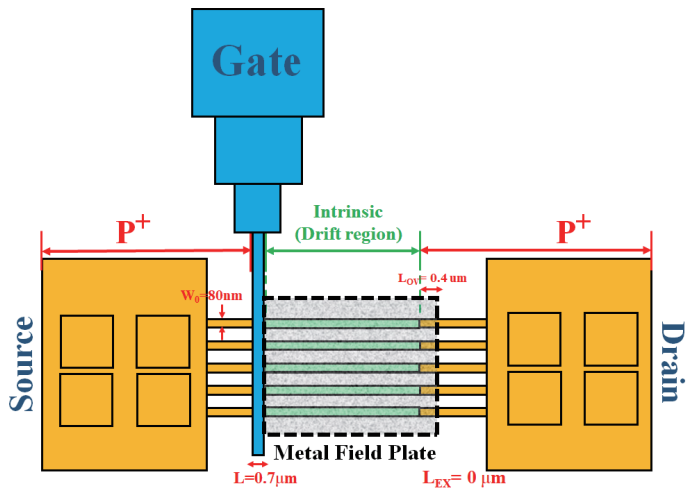

(a)

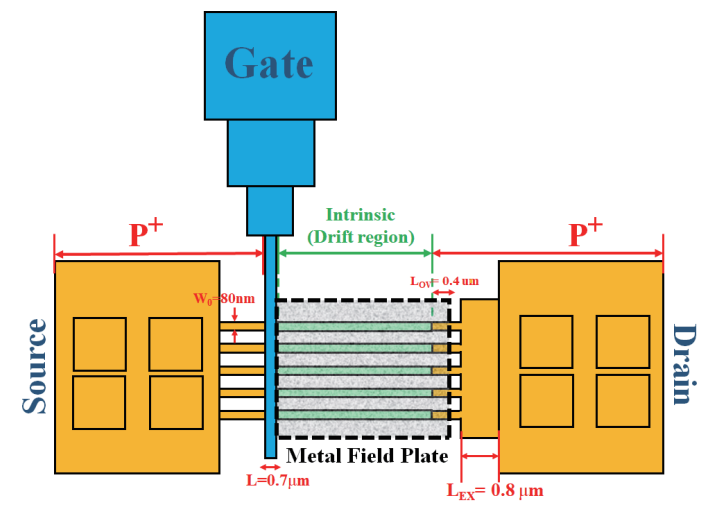

(b)

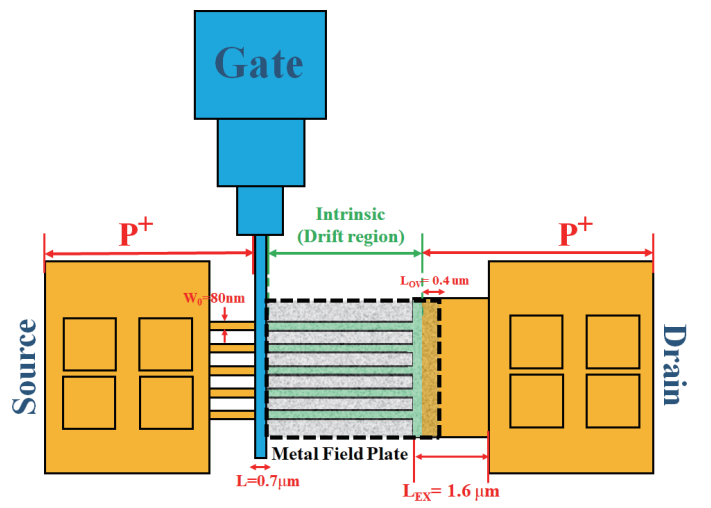

(c)

Fig. 1. (Color online) Schematic top view of poly-Si TFTs with (a) $L_{E X}=0 \mu \mathrm{m}$, (b) $L_{E X}=0.8 \mu \mathrm{m}$, and (c) $L_{E X}=$ $1.6 \mu \mathrm{m}$.

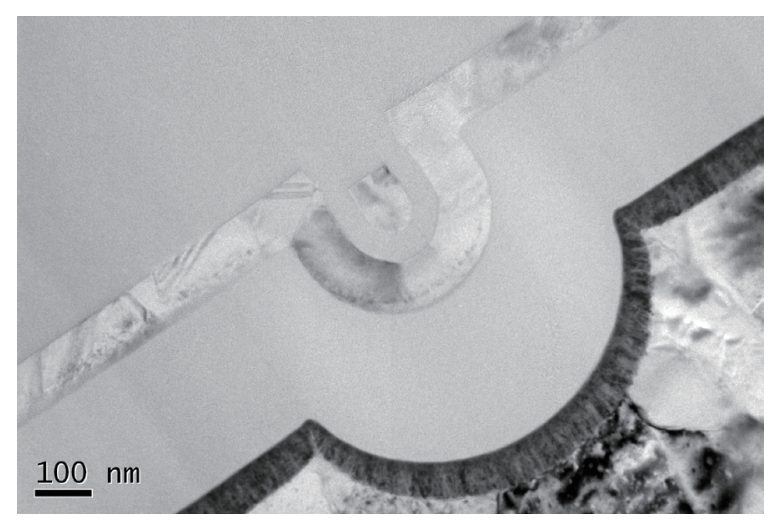

Fig. 2. TEM image of a single NW.

shows a cross-sectional transmission electron microscopy (TEM) image of a single NW. The bottom side of each channel wire $\left(W_{0}\right)$ is about $80 \mathrm{~nm}$. The measured width of each channel wire $\left(W_{n w}\right)$ is approximately $280 \mathrm{~nm}$ and the number of fins is five. Therefore, the effective channel width $W_{\text {eff }}$ is $14 \mathrm{~mm}$. All the poly-Si FinTFTs measured in this work have the same $W_{\text {eff. }}$ 


\subsection{Leakage current of the poly-Si FinTFTs with various $L_{E X}$}

Figure 3(a) shows the transfer characteristic curves of the poly-Si FinTFTs with various $L_{E X}$. The leakage currents of the poly-Si FinTFTs are clearly different for each sample and, as such, are governed by different leakage mechanisms. The leakage mechanisms for poly-Si FinTFTs are primarily derived from band-to-band tunneling (BTBT) and trap-assisted tunneling (TAT). In Fig. 3(a), the leakage current becomes larger as $L_{E X}$ increases from 0 to $0.8 \mathrm{~mm}$ and has a strong dependence on the gate voltage. As $L_{E X}$ increases, the increase in ion implantation area causes the generation of more traps and leads to a higher leakage current. In addition, increasing $L_{E X}$ can reduce the longitudinal electric field at the intrinsic drift $/ \mathrm{N}+$ junction and improve the leakage current derived from L-BTBT. ${ }^{(9)}$ Therefore, the leakage current of the poly-Si FinTFTs with $L_{E X}=0.8 \mathrm{~mm}$ being higher than those with $L_{E X}=0 \mathrm{~mm}$ is mainly due to the larger number of traps resulting from ion implantation. When $L_{E X}$ increases to $1.6 \mathrm{~mm}$, the leakage current is higher at a low gate voltage than for the other values of $L_{E X}$ and shows a weak dependence on the gate voltage. The results indicate that TAT rather than BTBT dominates the leakage current of the poly-Si FinTFTs with $L_{E X}=1.6 \mathrm{~mm}$. To improve the electrical characteristics of the poly-Si TFTs, MWA at $1.2 \mathrm{~kW}$ for $100 \mathrm{~s}$ in $\mathrm{N}_{2}$ ambient instead of forming gas annealing is carried out as a post-metallization annealing (PMA) process [shown in Fig. 3(b)]. After PMA, in contrast, the leakage current decreases as $L_{E X}$ increases from 0 to $0.8 \mathrm{~mm}$, which is attributed to the reduction in interface trap density. Therefore, the leakage current of the poly-Si FinTFTs with $L_{E X}=0.8 \mathrm{~mm}$ being lower than that of the FinFETs with $L_{E X}=0 \mathrm{~mm}$ is mainly due to the longitudinal electric field at the intrinsic drift $/ \mathrm{N}+$ junction.

\subsection{Leakage current of the poly-Si FinTFTs at different temperatures}

To investigate the leakage mechanisms of poly-Si FinTFTs with various $L_{E X}$, transfer characteristic curves obtained at various temperatures are compared. Figure 4 shows the poly-Si FinTFTs with $L_{E X}=0.8 \mathrm{~mm}$ at $25,50,75$, and $100{ }^{\circ} \mathrm{C}$ under drain voltages of -1 and $-3 \mathrm{~V}$.

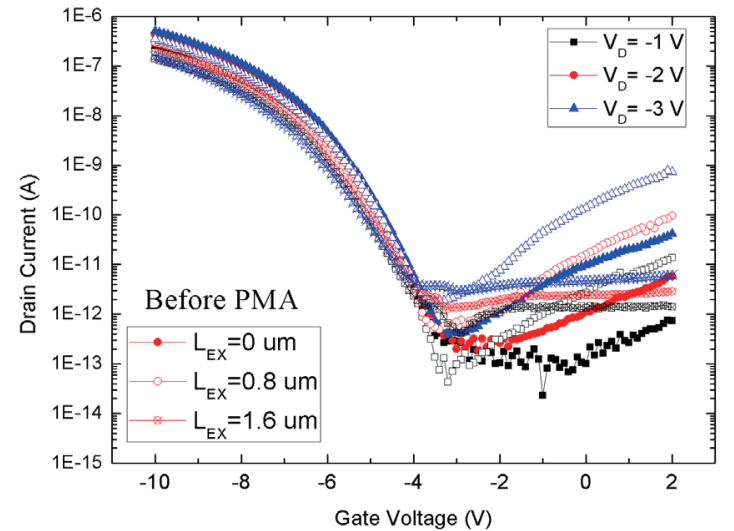

(a)

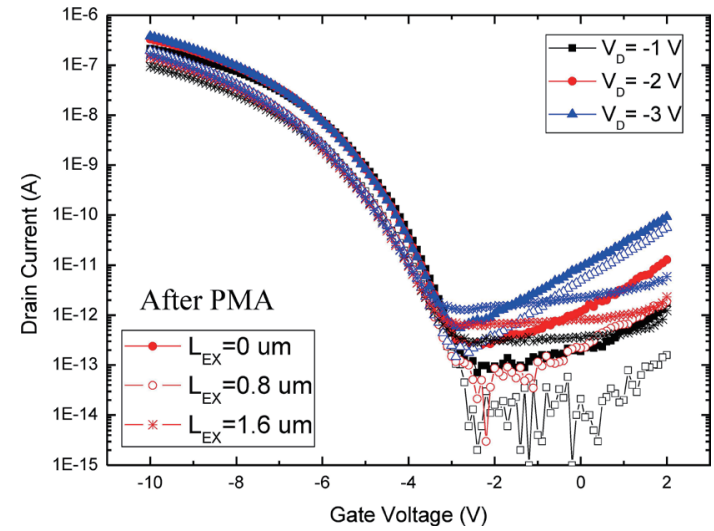

(b)

Fig. 3. (Color online) Measured transfer characteristic curves of the poly-Si FinTFTs with various $L_{E X}$ (a) before and (b) after PMA. 
The dependence of leakage current on temperature becomes less sensitive with increasing drain voltage. The bandgap change is small and decreases with increasing temperature. When the temperature is increased, there is a small bandgap change, resulting in a small increase in BTBT current. Therefore, the BTBT becomes dominant as the drain voltage increases.

Figure 5 shows the poly-Si FinTFTs with $L_{E X}=0$ and $1.6 \mathrm{~mm}$ at a drain voltage of $-3 \mathrm{~V}$ at various temperatures. When the drain voltage is fixed at $-3 \mathrm{~V}$, the temperature dependence of the leakage current is stronger for the poly-Si FinTFTs with $L_{E X}=1.6 \mathrm{~mm}$ than for those with $L_{E X}=0$ and $0.8 \mathrm{~mm}$ (as shown in Figs. 4 and 5). However, the drain voltage dependence of the leakage current is weaker for the poly-Si FinTFTs with $L_{E X}=1.6 \mathrm{~mm}$ than for the others. These results confirm that TAT rather than BTBT dominates the leakage current of the poly-Si FinTFTs with $L_{E X}=1.6 \mathrm{~mm}$. In addition, the temperature dependence of the leakage current becomes weak when the poly-Si FinTFTs undergo the PMA treatment. This result demonstrates that the trap density for the poly-Si FinTFTs is reduced by the PMA treatment.

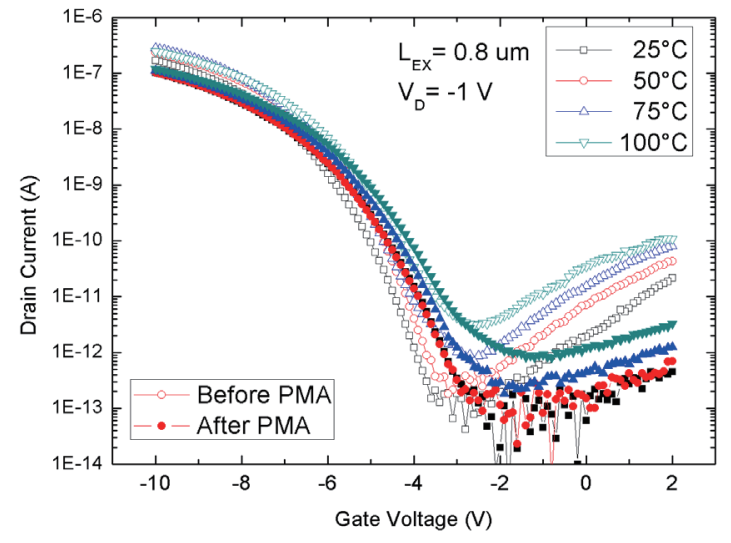

(a)

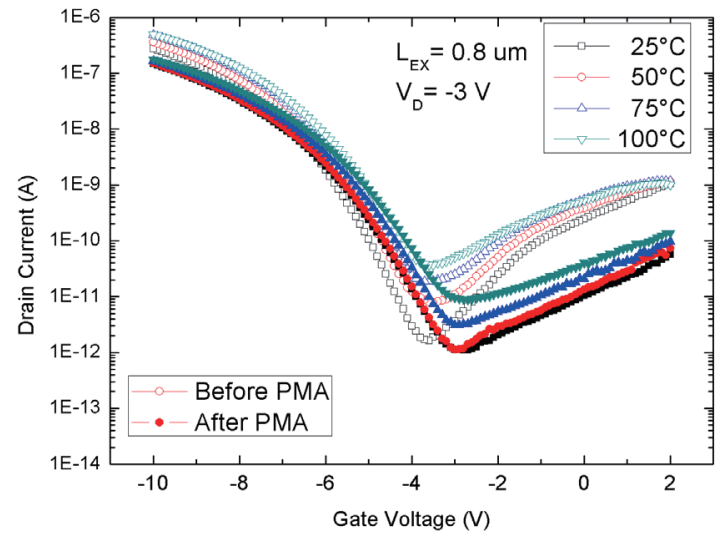

(b)

Fig. 4. (Color online) Measured transfer characteristic curves of poly-Si FinTFTs with $L_{E X}=0.8 \mu \mathrm{m}$ at various temperatures at (a) $V_{D}=-1 \mathrm{~V}$ and (b) $V_{D}=-3 \mathrm{~V}$.

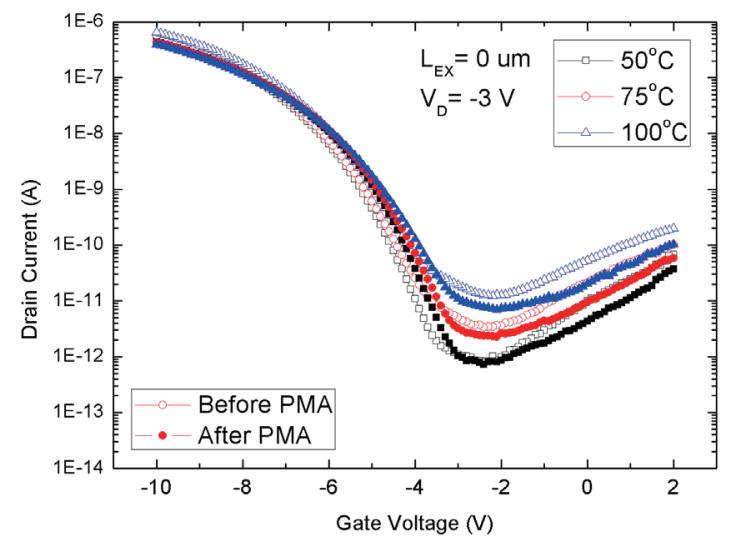

(a)

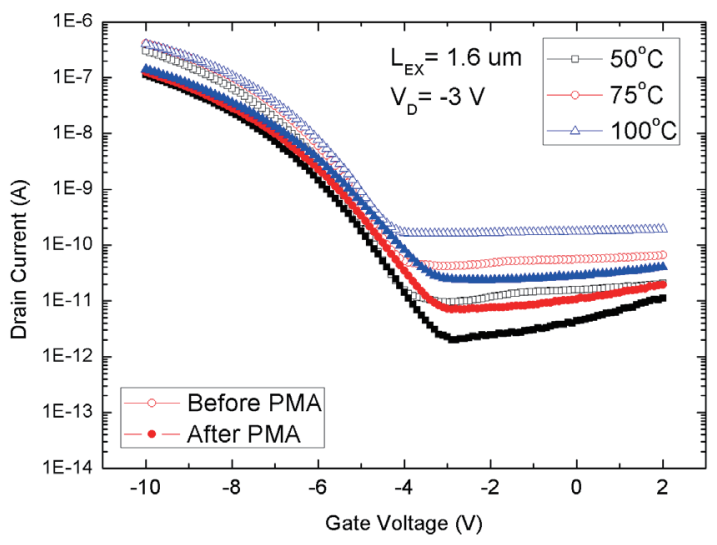

(b)

Fig. 5. (Color online) Measured transfer characteristic curves of poly-Si FinTFTs at $V_{D}=-3 \mathrm{~V}$ at various temperatures with (a) $L_{E X}=0 \mu \mathrm{m}$ and (b) $L_{E X}=1.6 \mu \mathrm{m}$. 


\section{Conclusions}

The off leakage current of poly-Si FinTFTs with different $L_{E X}$ has been investigated. After PMA, the leakage current decreases as $L_{E X}$ increases, especially at a high gate voltage and a high drain voltage. For a small $L_{E X}(=0$ and $0.8 \mathrm{~mm})$, the dependence of leakage current on temperature is less sensitive, which indicates that the BTBT is dominant. The BTBT is primarily dependent on drain voltage rather than on temperature. As $L_{E X}$ increases to 1.6 $\mathrm{mm}$, the weak dependence of leakage current on drain voltage and the strong dependence of leakage current on temperature indicate that TAT is the dominant leakage mechanism, where an increase in $L_{E X}$ can reduce the longitudinal electric field and L-BTBT. These results provide an effective method of suppressing the GIDL current of FinFETs in which L-BTBT instead of T-BTBT is the primary source for 3D transistors such as FinFETs.

\section{Acknowledgments}

This work was supported by the Ministry of Science and Technology of Taiwan under Contract MOST 108-2221-E-027-048, and in part by the National Nano Device Laboratories, Taiwan. The authors would like to thank the National Nano Device Laboratories for technical support.

\section{References}

1 J. M. Lee, K. H. Cho, D. W. Kim, and I. Chung: Jpn. J. Appl. Phys. 55 (2016) 04ED17. https://doi.org/10.7567/ JJAP.55.04ED17

2 S. Sahay and M. J. Kumar: IEEE Trans. Electron Devices 64 (2017) 3007. https://doi.org/10.1109/ TED.2017.2702067

3 J. Fan, M. Li, X. Xu, Y. Yang, H. Xuan, and R. Huang: IEEE Trans. Electron Devices 62 (2015) 213.

4 S. Sahay and M. J. Kumar: IEEE Trans. Electron Devices 63 (2016) 5055.

5 J. Hur, B. H. Lee, M. H. Kang, D. C. Ahn, T. Bang, S. B. Jeon, and Y. K. Choi: IEEE Electron Device Lett. 37 (2016) 541.

6 S. Sahay and M. J. Kumar: IEEE Trans. Electron Devices 64 (2017) 2604.

7 S. Rewari, V. Nath, S. Haldar, S. S. Deswal, and R. S. Gupta: IEEE Trans. Electron Devices 65 (2018) 3.

8 A. K. Jain, S. Sahay, and M. J. Kumar: IEEE J. Electron Devices Soc. 6 (2018) 611. https://doi.org/10.1109/ JEDS.2018.2829633

9 H. H. Hu, Y. W. Zeng, and K. M. Chen: Appl. Sci. 8 (2018) 1406.

10 S. Y. Kim, W. F. Loke, B. Jung, and K. Roy: IEEE Trans. Electron Devices 59 (2012) 2296. https://doi. org/10.1109/TED.2012.2202238

11 H. Yin, W. Xianyu, A. Tikhonovsky, and Y. S. Park: IEEE Trans. Electron Devices 55 (2008) 578. https://doi. org/10.1109/TED.2007.912364

12 Y. C. Wu, C. Y. Chang, T. C. Chang, P. T. Liu, C. S. Chen, C. H. Tu, H. W. Zan, Y. H. Tai, and S. M. Sze: Proc. IEEE Int. Electron Devices Meeting (IEDM) (2004) 777. https://doi.org/10.1109/IEDM.2004.1419289

13 M. S. Yeh, Y. J. Lee, M. F. Hung, K. C. Liu, and Y. C. Wu: IEEE Trans. Nanotechnol. 12 (2013) 636. https:// doi.org/10.1109/TNANO.2013.2265778

14 Y. J. Lee, Y. L. Lu, F. K. Hsueh, K. C. Huang, C. C. Wan, T. Y. Cheng, M. H. Han, J. M. Kowalski, J. E. Kowalski, D. Heh, H. T. Chuang, Y. Li, T. S. Chao, C. Y. Wu, and F. L. Yang: Proc. IEEE Int. Electron Devices Meeting (IEDM) (2009) 31. http://doi.org/10.1109/IEDM.2009.5424426

15 T. L. Alford, D. C. Thompson, J. W. Mayer, and N. D. Theodore: J. Appl. Phys. 106 (2009) 114902-1. https:// doi.org/10.1063/1.3260245 


\section{About the Authors}

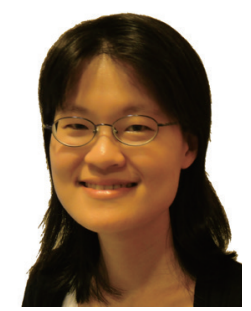

Hsin-Hui Hu received her M.S. and Ph.D. degrees in electronics engineering from National Chiao Tung University, Hsinchu, Taiwan, in 2004 and 2008, respectively. She is currently an associate professor with the Department of Electronic Engineering, National Taipei University of Technology, Taipei, Taiwan. Her current research interests include radio frequency, high-voltage, and nanoelectronic devices.

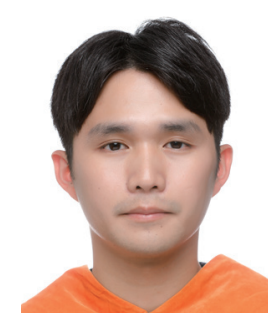

Chun-Lin Huang received his M.S. degree in electronic engineering from the National Taipei University of Technology, Taipei, Taiwan, in 2019.

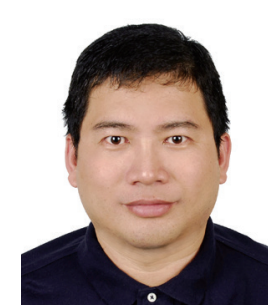

Yao-Jen Lee was born in Kaohsiung, Taiwan, in 1976. He received his Ph.D. degree from the Institute of Electronics, National Chiao Tung University, Hsinchu, Taiwan, in 2004. He is currently a research fellow with the National Nano Device Laboratories, Hsinchu, and also with the Department of Physics, National Chung Hsing University, Taichung, Taiwan.

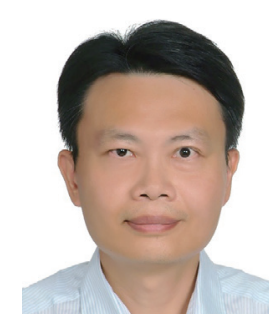

Kun-Ming Chen received his M.S. and Ph.D. degrees in electronics engineering from National Chiao Tung University, Hsinchu, Taiwan, in 1996 and 2000, respectively. In 2000, he joined the National Nano Device Laboratories, Hsinchu, Taiwan, as an associate researcher, where he became a research fellow in 2007. His current research interests include the fabrication and characterization techniques of high-frequency and high-power transistors. 